\title{
Effect of early versus delayed feeding following emergency endoscopic therapy for acute esophageal variceal bleeding on short-term outcomes Tarek Goda ${ }^{\mathrm{a}}$, Abdelrahman Mokhtar ${ }^{\mathrm{b}}$, Rokaya Anwar ${ }^{\mathrm{b}}$, Hazem Hakim ${ }^{\mathrm{b}}$, Ali Eleraki ${ }^{\mathrm{b}}$
}

Departments of ${ }^{\mathrm{a} C}$ Critical Care, ${ }^{\mathrm{b}}$ Hepatology and Gastroenterology, Faculty of Medicine, Mansoura University, Mansoura, Egypt

Correspondence to Hakim Hazem, MD of Internal Medicine, Assistant Professor of Hepatology and Gastroenterology Department, Faculty of Medicine, Mansoura University, Mansoura, 35516, Egypt

Tel: +201100100066;

e-mail: hzhzhkhk@yahoo.com

Received 14 April 2018

Accepted 9 July 2018

The Egyptian Journal of Internal Medicine 2018, 30:110-114

\begin{abstract}
Background/aim
It is generally assumed that systematic fasting is obligatory in the context of acute variceal bleeding owing to the fear of increasing the risk of rebleeding if patients instituted feeding early after endoscopy. However, this assumption is generally built on experts' beliefs instead of being based on large clinical trials. Such lack of clinical trials was the reason why we conducted this controlled study.

Patients and methods

Patients were selected from those with acute esophageal variceal bleeding admitted at the Hematemesis Unit at Mansoura Emergency Hospital. The study involved 90 patients who divided into two equal groups: group I included 45 patients as the early-feeding group and group II included 45 patients as the late-feeding group.

\section{Results}

There was no statistically significant difference regarding treatment complications distribution between the studied groups. The successful hemostatic rate was $100 \%$ in both groups. Neither rebleeding incidents nor deaths were encountered in the first 5 days in either group.

\section{Conclusion}

Early feeding with a liquid diet after successful endoscopic therapy of bleeding esophageal varices did not enhance rebleeding or increase mortality in the first 5 days following endoscopic intervention.
\end{abstract}

\section{Keywords:}

early feeding, esophageal varices, rebleeding

Egypt J Intern Med 30:110-114

(c) 2018 The Egyptian Journal of Internal Medicine

1110-7782

\section{Introduction}

One of the serious complications of cirrhosis is the development of gastroesophageal varices. It is estimated that esophageal or gastric varices are present in almost half of all patients at the time of diagnosis of cirrhosis, and they develop at a rate of $7 \%$ per year [1]. Although previous studies have repeatedly shed light on the issues of employment of vasoconstrictors, endoscopic modality, and antibiotics in the management of acute variceal bleeding, they rarely discussed the role of nutrition in the management of acute variceal bleeding.

\section{Aim}

The aim was to compare the effect of early versus delayed feeding on the incidence of rebleeding encountered in the first 5 days after endoscopic control of acute esophageal variceal bleeding via band ligation or injection sclerotherapy.

\section{Patients and methods}

This prospective study took place from July 2016 to August 2017, at Hematemesis Unit, Mansoura
Emergency Hospital. It involved 90 patients with liver cirrhosis presented with acute esophageal variceal bleeding proven by endoscopy within $12 \mathrm{~h}$ from admission.

\section{Inclusion criteria}

The inclusion criteria were as follows:

(1) Age greater than 18 years old.

(2) Patients with cirrhotic portal hypertension diagnosed by history, physical examinations, liver histology, or radiology.

(3) Patients presented with acute esophageal variceal bleeding.

(4) Successful control of variceal bleeding.

(5) Patients' consciousness level allows safe oral intake. 


\section{Exclusion criteria}

The following were the exclusion criteria:

(1) Association with a severe systemic illness, such as sepsis, uremia or congestive heart failure

(2) Advanced hepatocellular carcinoma (HCC) stage greater than or equal to Barcelona Clinic Liver Cancer C.

(3) Failure to control esophageal bleeding by emergency endoscopic therapy.

(4) Bleeding gastric varices.

(5) Child-Pugh score greater than or equal to 13.

(6) Refusal to participate.

(7) Uncooperative patients.

\section{Ethical concerns}

Written informed consents were obtained from the patients or their relatives participating in this study after informing them about the steps of the study. The study was approved by the Ethical Committee in Faculty of Medicine Mansoura University.

\section{Methods}

Between July 2016 and August 2017, 90 patients hospitalized with features of acute esophageal variceal hemorrhage confirmed by emergency upper gastrointestinal (GI) endoscopy were considered eligible for analysis in our study. Endoscopy was carried out within $12 \mathrm{~h}$ after hospitalization except in patients with hepatic encephalopathy where endoscopy was delayed till their consciousness improved. Similarly, balloon tamponade was employed in patients presenting with massive hematemesis and hemodynamic instability despite resuscitation to arrest the bleeding till patients' clinical condition stabilized and permitted carrying out endoscopy. After achieving successful hemostatic control of bleeding esophageal varices via endoscopic variceal ligation (EVL) or endoscopic injection sclerotherapy (EIS), the eligible participants were then randomized to one of two equal groups: (a) the early-feeding group and (b) the delayed-feeding group.

\section{Early-feeding group}

We instructed patients in this group to start oral feeding just $4 \mathrm{~h}$ after undergoing emergency endoscopic intervention. Regarding the type of the diet commenced, patients in this group were allowed only a liquid diet such as milk, fruit juice, or rice in liquid formula in the first $48 \mathrm{~h}$. Solid foods were not permitted. In the following 3 days, they were allowed to start a soft diet, and then starting from the sixth day from endoscopy, they were provided a regular diet.

\section{Delayed-feeding group}

Regarding participants in this group, we instructed them to completely withhold feeding for 2 days after emergency endoscopic management. Regarding nutritional supply during that period, we provided them with $1500 \mathrm{ml} /$ day parenteral fluids ( $0.9 \%$ saline and dextrose water) with adequate electrolytes, and this continued for $48 \mathrm{~h}$. Following 2 days of abstaining from eating, patients were commenced on a liquid diet for 1 day, and afterwards, we provided them with a soft diet for the following 2 days, and eventually they resumed a regular diet on the sixth day following emergency upper GI endoscopy.

Both groups continued to receive vasoconstrictor therapy in the form of terlipressin or octereotide for 3 days and prophylactic antibiotics (ceftriaxone or cefotaxime) for 5 days following endoscopy. Blood transfusions along with fresh frozen plasma (FFP) and platelets were given when indicated. Upon participation in our study, patients in both groups underwent abdominal ultrasonography to identify those presenting with ascites or HCC. Regarding the primary end points in our study, this included rates of successful initial hemostasis as well as early rebleeding within the first 5 days. Meanwhile, the secondary end points included new development of ascites (or worsening of the degree of preexistent ascites), rebleeding rate and mortality within the first 6 weeks.

\section{Statistical analysis}

Data entry and statistical analyses were performed using SPSS (statistical package of social sciences) version 20.0 (SPSS Inc., Chicago, IL, USA). Parametric data were expressed in mean \pm standard deviation. Non parametric data were expressed in median, minimum and maximum. Normality of data was first tested by one sample Kolmogrov-Smirnov test. In addition, independent $t$ test was used to compare means for continuous parametric variables of each two different groups. Also, Mann-Whitney $U$ test (z) was used to compare non parametric continuous variables in two different groups. In addition, Pearson Chi-square tests were used to compare the categorical variables between the two groups.

\section{Results}

This study was conducted on 90 patients with liver cirrhosis presented with acute variceal bleeding. These patients were randomized into two equal groups: 45 patients in the early-feeding group and the other 45 in the delayed-feeding group. 
At time of admission, both early-feeding and delayedfeeding groups were similar in their baseline clinical data such as the severity of liver cirrhosis as demonstrated by Child-Pugh score, grading of esophageal varices, percentage of active bleeding, and the usage of vasopressors. Following enrollment, four $(8.9 \%)$ patients in the early-feeding group in addition to three $(6.7 \%)$ patients in the delayed feeding group presented with massive hematemesis and became hemodynamically unstable, which necessitated the employment of balloon tamponade to arrest the bleeding while resuscitating the patients till their status permitted endoscopic intervention.

The main outcomes are shown in Tables 1 and 2. EVL was the modality used in most patients in both groups compared with EIS, which was only used in four patients in both groups. Treatment failure or failure in the control of acute bleeding was not encountered in either group. However, we encountered two rebleeding episodes in two patients in the early-feeding group; both were Child-Pugh class C. The first patient developed severe early rebleeding on the seventh day and died after failure of resuscitation whereas the second one rebled on the 20th day after endoscopy and survived following adequate resuscitation. Meanwhile, in the delayed feeding group, only one

Table 1 Baseline data of both groups

\begin{tabular}{|c|c|c|c|}
\hline & $\begin{array}{l}\text { Early-feeding } \\
\text { group }(n=45)\end{array}$ & $\begin{array}{l}\text { Delayed- } \\
\text { feeding group } \\
(n=45)\end{array}$ & $P$ \\
\hline Age (years) & $57.56 \pm 8.7$ & $56.96 \pm 8.8$ & 0.75 \\
\hline Male/female & $36 / 9$ & $32 / 13$ & 0.46 \\
\hline Rural/urban & $31 / 14$ & $26 / 19$ & 0.27 \\
\hline Ascites & 12 & 11 & 0.81 \\
\hline $\mathrm{HCC}$ & 6 & 3 & 0.48 \\
\hline SBP & 3 & 2 & 1 \\
\hline Hepatic encephalopathy & 2 & 4 & 0.67 \\
\hline Albumin $(\mathrm{g} / \mathrm{dl})$ & $2.76 \pm 0.5$ & $2.79 \pm 0.6$ & 0.79 \\
\hline Bilirubin (mg/dl) & $2.32 \pm 1.0$ & $2.3 \pm 0.9$ & 0.68 \\
\hline Hemoglobin (g/dl) & $8.8(5-14)$ & $8(4-13)$ & 0.59 \\
\hline $\begin{array}{l}\text { Child-Pugh class } \\
\text { (A/B/C) }\end{array}$ & $9 / 25 / 11$ & $10 / 24 / 11$ & 0.9 \\
\hline $\begin{array}{l}\text { Attack of upper GI } \\
\text { bleeding (first/recurrent) }\end{array}$ & $22 / 26$ & $26 / 19$ & 0.39 \\
\hline Previous NSBB intake & 21 & 15 & 0.19 \\
\hline Terlipressin/octreotide & $31 / 14$ & $26 / 19$ & 0.27 \\
\hline $\begin{array}{l}\text { Balloon tamponade } \\
\text { before endoscopy }\end{array}$ & 4 & 3 & 0.69 \\
\hline $\begin{array}{l}\text { Size of esophageal } \\
\text { varices (F1/F2/F3) }\end{array}$ & $4 / 14 / 27$ & $3 / 13 / 29$ & 0.88 \\
\hline $\begin{array}{l}\text { Active bleeding during } \\
\text { endoscopy }\end{array}$ & 13 & 16 & 0.49 \\
\hline $\begin{array}{l}\text { Blood transfusion units } \\
\text { (minimum-maximum) }\end{array}$ & $2(0.0-5.0)$ & $2(0.0-5.0)$ & 0.48 \\
\hline
\end{tabular}

Gl, gastrointestine; HCC, hepatocellular carcinoma; NSBB, nonselective $\beta$-blocker; SBP, systolic blood pressure. patient developed early rebleeding on the 10th day and he underwent a successful EVL and survived. Regarding mortality, two deaths were encountered in the early-feeding group: the first one died because of severe upper GI bleeding as mentioned earlier, whereas the second death was not related to bleeding varices but instead owing to septic shock, which was encountered 1 month after endoscopy. On the contrary, only one death was encountered in the late-feeding group, 3 weeks following endoscopy owing to septic shock.

\section{Discussion}

Acute variceal hemorrhage in patients with cirrhosis is a life-threatening condition with a predictable mortality rate of $\sim 20 \%$ at 6 weeks. Even after successful management of the initial bleeding, there remains a major risk of rebleeding, usually witnessed during the first 5 days and almost always before 11 days [2].

Patients with active variceal bleeding are generally predisposed to have an appreciably high incidence of early rebleeding $[3,4]$. Predictors of rebleeding or increased mortality include lower level of hematocrit, presence of portal vein thrombosis, high serum bilirubin and low albumin levels, presence of hepatic encephalopathy, HCC, model for end-stage liver disease score, Child-Pugh score, hepatic venous pressure gradient (HVPG) greater than or equal to $20 \mathrm{mmHg}$, development of infections, endoscopic appearance (active bleeding and clot on varix), and shock $[5,6]$.

The accurate timing of feeding after successful control of upper GI bleeding by EVL or EIS is an important subject that has rarely been explored. It is generally assumed that systematic fasting is obligatory in the context of arginine vasopressin (AVP) owing to the fear of increasing the risk of rebleeding if patients instituted feeding early on after endoscopy [7]. Although

Table 2 Main outcomes in the two treatment groups

\begin{tabular}{lccc}
\hline & $\begin{array}{c}\text { Early-feeding } \\
\text { group }(n=45)\end{array}$ & $\begin{array}{c}\text { Delayed-feeding } \\
\text { group }(n=45)\end{array}$ & $P$ \\
\hline EVL/EIS & $41 / 4$ & $41 / 4$ & 1 \\
Treatment failure & 0.0 & 0.0 & - \\
$\begin{array}{l}\text { Very early } \\
\text { rebleeding }\end{array}$ & 0.0 & 0.0 & - \\
Rebleed between 6 & 2 & 1 & 1 \\
and 42 days & & & \\
Mortality (days) & & & \\
$\quad$ First 5 & 0.0 & 0.0 & - \\
$\quad 6-42$ & 2 & 1 & 1 \\
\hline
\end{tabular}

EIS, endoscopic injection sclerotherapy; EVL, endoscopic variceal ligation. 
withholding feeding seems to be safe and carries less risk of early rebleeding, prolonged starvation is unethical to low-risk patients or those exposed to high level of catabolism like patients with cirrhosis who do not tolerate extended periods of starvation that can lead to protein breakdown and malnutrition in addition to hypoalbuminemia with subsequent worsening of ascites in these patients. Moreover, the assumption to withhold feeding following endoscopic control of variceal bleeding is generally built on experts' beliefs instead of being based on large clinical trials. Such lack of clinical trials was the reason why we conducted this controlled study.

Our study included 90 cirrhotic patients with acute variceal bleeding, both active bleeding and inactive bleeding at endoscopy. Our results revealed that early feeding with a liquid diet as early as $4 \mathrm{~h}$ after endoscopic therapy did not enhance rebleeding as compared with patients with delayed feeding for $48 \mathrm{~h}$. The current study showed that EVL is highly effective in the control of esophageal varices. After a successful endoscopic therapy, failure to control acute bleeding as well as very early rebleeding was not encountered in both early-feeding and delayed-feeding groups.

Only a couple of studies have evaluated enteral nutrition in patients with cirrhosis with bleeding from esophageal varices. Borderie et al. [8] randomly enrolled 12 patients for early enteral nutrition and 10 patients for fasting for 3 days following endoscopic treatment. Recurrent bleeding occurred in three patients of the enteral nutrition group, but in none of the patients of the control group. Owing to a small sample size (i.e. only 22 patients were enrolled), no statistical significant difference was reached. However, they concluded that enteral feeding may have an unfavorable effect on the short-term hemostasis of bleeding varices and it should be delayed. Recently, Lo et al. [9] conducted a study to determine whether early versus delayed feeding following EVL can improve hemostatic outcomes of patients with acute esophageal variceal bleeding. They randomized 36 patients into an early-feeding group after fasting for $4 \mathrm{~h}$ following EVL and 34 patients into a delayedfeeding group after fasting for $48 \mathrm{~h}$ following EVL, and found that very early rebleeding was not encountered in both groups. The authors concluded that early feeding with a liquid diet after successful endoscopic therapy of bleeding varices did not affect hemostasis, but it can reduce the duration of hospital stays.

Endoscopic findings in our study slightly differed from those in the study conducted by Lo et al. [9]. Although they included bleeding gastric varices, we excluded them from ours as our aim of the study was to focus on the effect of feeding on bleeding esophageal varices only. Moreover, in our study, eight patients, that is, four (8.9\%) in the early-feeding group and another four (8.9\%) in the delayed-feeding group, underwent EIS, whereas the rest of patients in both groups underwent EVL. This was not the case with the aforementioned study where all patients underwent EVL and none of them required EIS. This is attributed to the fact that these patients had already undergone chronic EIS in several endoscopic sessions which decreased the size of the varices and this rendered EVL not suitable for them. In our study, F3 varices had the upper hand in both earlyfeeding and delayed-feeding groups unlike the study performed by Lo et al. [9] where F2 varices had the upper hand. In our study, the grading of the varices in the early group was F1-F2-F3: 4-14-27 compared with the early group in the other study, which showed a different grading (F1-F2-F3: 6-22-8). Meanwhile, the grading of severity of the varices in the delayed-feeding group in our study was F1-F2-F3: 3-13-29 compared with F1-F2F3: 5-22-7 in the other study. The reason why the varices were more risky and their size was larger in our study may be largely owing to co-existence of bilharzial periportal fibrosis beside after hepatitic cirrhosis, as bilharziasis, similarly to $\mathrm{HCV}$, is still endemic in our locality as demonstrated in the study conducted by Haggag et al. [10] especially in the rural areas where the majority of our patients come from. Another reason is the inadequate portal hypertension control among our patients in terms of prescribing suboptimal doses of $\beta$-blockers and lack of patients' compliance and the follow-up endoscopies, which in turn resulted in progressive increase in portal pressure, with worsening of the degree of portal hypertension and subsequent development of risky large varices.

Based on our study, initiating oral feeding with a liquid diet as early as $4 \mathrm{~h}$ after endoscopy was similar to delayed feeding in terms of very early rebleeding and mortality in the first 5 days. The clinical outcomes regarding rebleeding rates, mortality, and adverse events were similar in both groups. This is in agreement with Lo et al. [9] who further reported that patients with acute variceal bleeding should be encouraged to eat as early as possible after successful EVL or EIS as adopting such policy has the benefits of improving patients' willingness to eat, supplementation of greater amount of calories, shorter duration of hospitalization, and a probable reduction in ascites development.

We did not measure the portal pressure in our study, and thus, we do not know for sure whether early feeding 
resulted in an increase in portal pressure or not. However, there was no statistically significant difference in terms of unfavorable outcomes between both the early-feeding and delayed-feeding groups, which reflects that earlyfeeding effect on mesenteric blood flow was not translated into bad clinical outcomes.

There are some limitations to our trial. The available sample size may be insufficient to demonstrate statistically significant differences between the studied groups. Moreover, a number of aspects such as the amount of calories supplied and the quantity of liquids ingested were not evaluated in our study.

In conclusion, our trial revealed that, in the setting of acute variceal bleeding, initiating feeding using a liquid diet just $4 \mathrm{~h}$ following institution of successful hemostasis via EVL or EIS did not have a negative effect on the hemostatic outcome in those patients and that early feeding did not enhance rebleeding or increase mortality in the first 5 days following endoscopic intervention.

\section{Financial support and sponsorship \\ Nil.}

\section{Conflicts of interest}

There are no conflicts of interest.

\section{References}

1 Kapoor A, Dharel N, Sanyal AJ. Endoscopic diagnosis and therapy in gastroesophageal variceal bleeding. Gastrointest Endosc Clin 2015; 25:491-507.

2 Burroughs AK, Triantos CK, O'beirne J, Patch D. Predictors of early rebleeding and mortality after acute variceal hemorrhage in patients with cirrhosis. Nat Rev Gastroenterol Hepatol 2008; 6:72.

3 Lo GH. Management of acute esophageal variceal hemorrhage. Kaohsiung J Med Sci 2010; 26:55e67.

4 Garcia-Tsao G, Bosch J. Management of varices and variceal hemorrhage in cirrhosis. N Engl J Med 2010; 362:823e32.

5 Bambha K, Kim WR, Pedersen R, Bida JP, Kremers WK, Kamath PS, et al. Predictors of early re-bleeding and mortality after acute variceal haemorrhage in patients with cirrhosis. Gut 2008; 57:814-820.

6 Augustin S, Genescà J. Diagnostic and prognostic markers in liver cirrhosis. Dis Markers 2011; 31:119-120.

7 Hebuterne X, Vanbiervliet G. Feeding the patients with upper gastrointestinal bleeding. Curr Opin Clin Nutr Metab Care 2011; 14:197e201.

8 Borderie C, De Ledinghen V, Beau P, Mannant PR, Ripault MP, Silvain C, et al. Early feeding or enteral nutrition in patients with cirrhosis after bleeding from esophageal varices? A randomized controlled study. Dig Dis Sci 1997; 42:536e41.

9 Lo GH, Lin CW, Hsu YC. A controlled trial of early versus delayed feeding following ligation in the control of acute esophageal variceal bleeding. $J$ Chin Med Assoc 2015; 78:642e7.

10 Haggag AA, Rabiee A, Abd Elaziz KM, Gabrielli AF, Abdel Hay R, Ramzy RMR. Mapping of schistosoma mansoni in the Nile delta, Egypt: assessment of the prevalence by the circulating cathodic antigen urine assay. Acta Trop. 2017; 167:9-17. 